\title{
Lethal Autonomous Weapons:
}

\author{
Designing War Machines with Values
}

\begin{abstract}
Steven Umbrello*
Lethal Autonomous Weapons (LAWs) have becomes the subject of continuous debate both at national and international levels. Arguments have been proposed both for the development and use of LAWs as well as their prohibition from combat landscapes. Regardless, the development of LAWs continues in numerous nation-states. This paper builds upon previous philosophical arguments for the development and use of LAWs and proposes a design framework that can be used to ethically direct their development. The conclusion is that the philosophical arguments that underpin the adoption of LAWs, although prima facie insufficient, can be actualised through the proposed Value Sensitive Design (VSD) approach. Hence, what is proposed is a principled design approach that can be used to embed stakeholder values into a design, encourage stakeholder cooperation and coordination and as a result promote social acceptance of LAWs as a preferable future fact of war.
\end{abstract}

\section{Lethal Autonomous Weapons - A Divisive Innovation}

Like many technological innovations, one fermenting ground that has remained a hotbed of novel technologies has been the military sphere. The future of war has and remains closely related to the current technological revolution at large. In recent years, developments in robotics, sensor technologies, information and communication technologies, among others, have made their way into the development of autonomous weapons systems that aim to supplement, or all out replace human combatants on the battlefield. The latest targeting systems and mobile weapons platforms are able to observe battlefield conditions in real-time and make accurate, and potentially lethal, interventions without human command-and-control. Although yet to be employed fully-autonomously in the battlefield, they are nonetheless in development with the intention of use. ${ }^{1}$ Similarly, there currently exists no international governance framework that regulate the development and use of LAWs. What follows then is a significant probability that states that develop LAWs will use them, making the understanding of the various ethical, social and legal issues of great importance given their potential impacts.

These developments put a burden on both legislative bodies, as well as society at large to analyse, evaluate their stances, and to take actions on whether, why, and how LAWs could become part of, if not all out replace the current human-centered dynamics that characterise the current battlefield landscape.

International bodies must decide the benefits and costs of developing and employing LAWs and how they can be developed in light of current technical limitations as well as the ethical and legal issues that are implicated by the stakeholders involved. Beyond this, what is similarly required is a principled way for designers of LAWs to embed agreed-upon principles such as the Laws of War (LoW) and/or the Rules of Engagement (RoE) as well as coordinate design flows and strategies between different developing bodies and nation states. Examples would be determining how to translate values such as proportionality into technical design requirments.

The study of LAWs is a multifaceted one which implicates many types of technologies and often treads on blurred lines. They have garnered mass interest which also resulted in the formation of various organisations aimed at their study, and even outright

\section{DOI: 10.21552/delphi/2019/1/7}

* Steven Umbrello, Managing Director at the Institute for Ethics and Emerging Technologies and graduate student at the University of Turin, Italy. For correspondence: <steve@ieet.org>. The author builds on his own work (fn 3 ).

1 Ashley Kirk, 'What Are the Biggest Defence Budgets in the World?' The Telegraph (London, 27 October 2015) 
ban of LAWs such as the Campaign to Stop Killer Robots. ${ }^{2}$ These discussions tend to focus on the issues of abdicating human responsibility in war to robots as well as the current technical limitations that make such abdication worrisome. Although these issues are important, they tend to ignore benefits that also arise from the implementation of LAWs. If we bracket technical feasibility in favor of looking at the potential benefits of LAWs we can see obvious boons such as the reduction of war crimes, reduction of unnecessary violence and naturally the reduction of causalities as a whole. ${ }^{3}$ However, if we speculate a future in which technical feasibility is a given, what is still needed is a design framework that can conceptualise the values that are most important to the development of LAWs as well as one that can help designers to incorporate those values in a principled way.

Because of the ethical issues that arise with the abdication of kill control away from humans, what is required is a way to conceptualise design flows between nation states that are proactive and recursively self-improving like the technologies themselves, a holistic and integrated approach is offered here through the design framework of VSD. It offers a way to conceptualise stakeholders' values, provides a principled approach to conceptualising those values as design requirements as well as visualise new ways in which disparate stakeholder groups can be coordinated based on commonly held values. ${ }^{4}$

This paper focuses on the ethical issues of developing and using LAWs (Section II) and discusses how the VSD methodology can be used to begin to ameliorate these concerns (Section III). The conclusion of this paper shows that regardless of any interna-

2 F Sauer, 'Stopping 'Killer Robots': Why Now Is the Time to Ban Autonomous Weapons Systems' (Arms Control Association, October 2016) < https://www.armscontrol.org/ACT/2016_10/ Features/Stopping-Killer-Robots-Why-Now-Is-the-Time-to-Ban -Autonomous-Weapons-Systems> accessed 8 October 2017

3 Steven Umbrello, Phil Torres, Angelo F. De Bellis, 'The Future of War: The Ethical Potential of Leaving War to Lethal Autonomous Weapons' (2019) Al \& Society (forthcoming)

4 Steven Umbrello, Beneficial Artificial Intelligence Coordination by Means of a Value Sensitive Design Approach. Big Data Cogn. Comput. 2019, 3, 5

5 A Krishnan, Killer Robots : Legality and Ethicality of Autonomous Weapons (Ashgate, 2009); See also N E Sharkey, 'Grounds for Discrimination: Autonomous Robot Weapons' (2008) 11 RUSI Def Syst 2, 86

6 J Galliott, Military Robots: Mapping the Moral Landscape (Ashgate, 2014) tional regulation on how LAWs are constructed, certain values will nonetheless have to be codified and formalised in programming. VSD is argued to be one way for international bodies to actualise principled design flows and encourage social acceptance.

\section{Arguments for the Development of LAWs}

The debate regarding the development of LAWs has been heated, with both supporters and detractors forwarding convincing arguments for each of their cases. Detractors commonly cite the abdication of killcontrol as something that should remain always within meaningful human control. ${ }^{5}$ Similarly, technical limitations of discerning legitimate combatants from civilians and surrendering agents are often cited as sufficient for halting the implementation of LAWs on the battlefield. Supporters, on the other hand, tend to take a more consequentialist approach in evaluating the use of LAWs arguing that the total risk-calculus of combatants of the field is automatically reduced with the introduction of LAWs. As well as this cold logic being brute, it is also overly reductive, ignoring many of the more nuanced and convincing arguments for the adoption of LAWs. Arguments, for example, can also be made that the suffering caused by impressionable human psychology on the battlefield, such as war crimes, can also be abated if the total quantity of human combatants is reduced via their replacement with LAWs, albeit foreseeably only utilized by more affluent nation states that are economically capable of investing in their development and ultimate use. Similarly, more personal suffering such as PTSD that is suffered by both on the ground combatants as well as remote operators such as drone pilots can be abated through the deployment of fully autonomous systems. ${ }^{6}$ Regardless, both sides acknowledge current technological constraint and limitations, as well as the risks associated with the susceptibility of system architecture. Extensive evaluation and anticipatory analysis of the potential risks of LAWs are an essential part of a responsible research and design operation and thus both essential prerequisites for international acceptance as a legitimate means of engaging in warfare.

The allure of LAWs is evident in the US's use of 'unmanned aerial vehicles' (UAVs, also known as 'drones') in Iraq and Syria. These semi-autonomous 
systems offer a cheap, effective, and relatively precise means for conducting both surveillance and engaging enemy combatants. More generally speaking, the expanding use of UAVs in combat zones is consistent with the more general inclination toward high-precision weaponry and away from larger, more destructive weapons like those in the world's nuclear arsenals. And there may be good reason for their proliferation, for example: the use high-precision weapons like LAWs to achieve a state's military objectives could reduce the probability and proportion of indiscriminate harm, thus violating the laws of war and rules of engagement less than might otherwise have been possible. The 'ease-of-use' of LAWs that are fully autonomous could enhance the 'balance of terror' that prevents conflict from breaking out by providing a credible means for retaliation: 'If you strike me first, I will unleash a swarm of LAWs that devastate your infrastructure, poison your streams, set fire to your farms, destroy your armies, and assassinate your leaders."

Regardless of which side of the argument one finds themselves, the fact of the matter is that LAWs are currently in the development processes by multiple nation-states. What is needed is not an outright moratorium or ban - which will only drive research into ungovernable and unmonitorable depths - but rather an active, participatory and stakeholder central approach to international governance that can implement, at least, a set of design principles that defense departments and private contractors can use to design their LAWs with the values of the international community in mind. This does not entail of course that all LAWs will consequentially be developed with these principles, but what it does is bring LAWs under the umbrella of existing statutes that govern the use of conventional weapons at the international level. Contravention of these conventions come with them their prescribed punitive measures.

What is then required is a standardised design methodology that is able to translate the values that can be distilled from these international conventions (ie, Laws of War [LoW] and Rules of Engagement $[$ RoE] $)$ into actionable design requirements.

\section{Value Sensitive Design}

Value Sensitive Design (VSD) is one of many design frameworks that has been proposed as an approach to designing sociotechnical systems with values in mind. Originating from the field of human-computer interaction (HCI), VSD has since been re-formalised for application to other technologies beyond HCI such as energy systems, nanotechnologies, biotechnologies, robotics, and artificial intelligence systems. 5

VSD begins with the premise that technologies are not value-neutral tools, but rather value-laden with both explicit and implicit values. ${ }^{8}$ Because of this, the founders of VSD sought to develop a design approach that can guide design flows to incorporate the values of affected stakeholders. ${ }^{9}$ Instead of the conventional means of analysing the moral status of technologies, ie, how it is introduced, used, and understood in societal context, VSD aims to evaluate at the effect that technology has on the moral landscape, establish the values of stakeholders, and embed those values in the early design phases. The VSD approach, through its tripartite structure of conceptual, empirical and technical investigations aims to determine the values of stakeholders, envision how those values can be construed as design requirements and evaluate how those design requirements can be supported or constrained by the technology under questions. VSD is understood as a flexible and adaptable approach that can be adopted by a variety of designer groups across a range of design disciplines. In doing so, its ability to be integrated into ongoing practices by working teams makes its adoption by industry and designers more favorable. VSD doesn't aim to overturn the engineering practices of designers in such a way that requires new or demanding changes; instead, VSD is an approach that poses ways of modifying existent designing and engineering practices in such a way as to include stakeholder values.

The VSD methodology can also be drawn upon in order to help researchers and designers in complying with existent governance frameworks that aim to regulate the conduct of developers. Because there are currently no governance frameworks that govern

$7 \quad$ (n 3$) 3$

8 Values is defined in way that the originators of VSD defined it: The Oxford English Dictionary definition of this sense of value is: the principles or standards of a person or society, the personal or societal judgement of what is valuable and important in life.

9 B Friedman et al, 'Charting the Next Decade for Value Sensitive Design' (2015) 1 Aarhus Ser Hum Centered Comput 1, 4 
LAWs, bringing them under the statutes that govern conventional weapons use may be a good initial step. Similarly, programming LAWs not with complex moral law theories, but instead existing conventions such as the LoW and RoE are more than sufficient to govern battlefield actions. This would be one of the initial considerations in the conceptual investigations of the design of LAWs. Determining the values of stakeholders (in this case primarily military stakeholders) and beginning to conceptualise how those values can be translated into design requirements. Because work has already been done on what values are important and to whom, as well as unveiling the complexity of programming conventionally conceived of modern moral theories (utilitarianism, deontology, virtue ethics, etc), the proposal to begin by programming already-in-development LAWs with the LoW and RoE is a reasonable initial step. Because the LoW and RoE are governed at the international level, programming LAWs with these set of principles will bring them under, at least, an umbrella of international governance.

The second essential stage of VSD is that of empirical investigations. Current manufacturing practices and regulations must be taken into account, particularly their applicability to LAWs regarding their techniques and practices in design. Naturally, fields of overlap will emerge, and perhaps existent regulatory frameworks will be proportionate. However, LAWs implicate both robotics and AI, the latter of which is a converging technology and thus not only may current issues in manufacturing practices be intensified but also issues that arise from the overlap of the converging domains such as nanotechnologies, biotechnologies and information and communication technologies. The VSD approach allows for the values that emerge from this convergence to be accounted for during the design phases to cover the emerging ethical issues that may emerge. Hence, not only how LAWs affects other converging technologies must be taken into consideration, but also how these other technologies change LAWs. Because of this, the testing practices characterised under the empirical investigations stage of VSD aim to expand both the current and future testing practices of VSD as well as serve as a means of checking the strength

10 B Friedman and $\mathrm{H}$ Kahn Jr, 'Value sensitive design: Theory and methods' (2002) UW CSE Technical Report of the initial conceptual investigations and how those instantiated values map on to real applications. ${ }^{10}$

For the VSD approach to be successful in integrating the values of military stakeholders at the design phase, LAWs designers must take up the VSD approach and inform the framework in a way that makes it specifically tailored to their domain. This will involve LAWs researchers drawing from existing practices and assimilating them into the VSD approach. This augmentation of the VSD method, informed by the specific domain to which it has been applied, has been a subject of the VSD literature since its inception, and that literature can be levied to help LAWs researchers in determining the most potent way of quickly integrating the VSD into their current design practices in a seamless way.

Future research should engage with the VSD literature as well as case studies to establish the most practical policy frameworks that provide incentives and other tractable steps for the implementation of VSD in this capacity. Similarly, future research should purpose to consolidate investigations of current LAWs enterprises as well as what regulatory structures may emerge and how those may affect industries. This research is imperative given that doing otherwise may appear as a demand on venture capitalists, researchers, and military stakeholders involved in development to ignore very concrete profit motives, and market forces, and instead to spend time on abstract ethical principles that would (in their view) put them at a disadvantage to their competition in bringing products to market quickly, or putting them at a strategic disadvantage in terms of military capacity. Asking them to do that seems unlikely to achieve much. Hence, in order to more efficiently incorporate values in design via a VSD framework researchers need to engage in comprehensive and integrated vision analyses rather than solely ethical ones.

\section{Conclusions}

LAWs, whether we like it or not, are currently in development. The trend towards the use of automation and technology as a way to remove human lives from the battlefield suggest this. Technologies are always imbued with values, either implicitly or explicitly. Accordingly it is vital that we develop a system of international governance around LAWs. This paper pre- 
sented a simple measure that can be taken as a potential first step. By proposing the VSD designing approach coupled with explicit incorporation of the Laws of War and Rules of Engagement as a basis for determining design requirements, LAWs can be both designed with a consciousness of values in mind as well as be brought under international statues that govern convention weapons use as well as engagement rules. Future research projects should explore exactly what values are already being implicated in the design of LAWs both from domestic and international perspectives. Likewise, these projects should look at how international regulations can be used to implement and administer a design-for-values approach to the development and implementation of LAWs that account for technical constraints that can be used as an initial primer for future ethical considerations in design. 